Article

\title{
Dysregulated Iron Metabolism-Associated Dietary Pattern Predicts an Altered Body Composition and Metabolic Syndrome
}

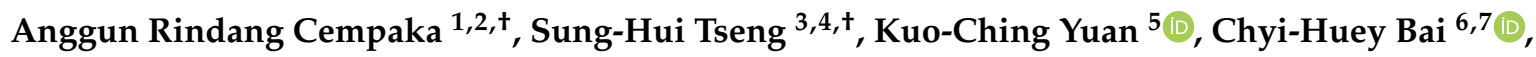 \\ Alexey A. Tinkov ${ }^{8,9,10}$ (D), Anatoly V. Skalny ${ }^{8,9,10}$ and Jung-Su Chang 2,11,12,13,*(D) \\ 1 Department of Nutrition Science, Faculty of Medicine, Brawijaya University, Malang 65145, Indonesia; \\ anggunrindangcempaka@gmail.com \\ 2 School of Nutrition and Health Sciences, College of Nutrition, Taipei Medical University, Taipei 110, Taiwan \\ 3 Department of Physical Medicine and Rehabilitation, Taipei Medical University Hospital, \\ Taipei 110, Taiwan; m003089010@tmu.edu.tw \\ 4 Department of Physical Medicine and Rehabilitation, School of Medicine, College of Medicine, \\ Taipei Medical University, Taipei 110, Taiwan \\ 5 Department of Emergency and Critical Care Medicine, Taipei Medical University Hospital, \\ Taipei 110, Taiwan; traumayuan@gmail.com \\ 6 Department of Public Health, College of Medicine, Taipei Medical University, Taipei 110, Taiwan; \\ baich@tmu.edu.tw \\ 7 Department of Public Health, College of Public Health, Taipei Medical University, Taipei 110, Taiwan \\ 8 Department of Medical Elementology, Peoples' Friendship University of Russia (RUDN University), \\ Moscow 117198, Russia; tinkov.a.a@gmail.com (A.A.T.); skalnylab@gmail.com (A.V.S.) \\ 9 Laboratory of Biotechnology and Applied Bioelementology, Yaroslavl State University, \\ Yaroslavl 150003, Russia \\ 10 Laboratory of Molecular Dietology, IM Sechenov First Moscow State Medical University, \\ Moscow 119146, Russia \\ 11 Graduate Institute of Metabolism and Obesity Sciences, College of Nutrition, Taipei Medical University, \\ Taipei 110, Taiwan \\ 12 Nutrition Research Center, Taipei Medical University Hospital, Taipei 110, Taiwan \\ 13 Chinese Taipei Society for the Study of Obesity (CTSSO), Taipei 11031, Taiwan \\ * Correspondence: susanchang@tmu.edu.tw; Tel.: +886-(2)-2736-1661 (ext. 6542); Fax: +886-(2)2737-3112 \\ + These authors contributed equally to this work.
}

Received: 19 October 2019; Accepted: 6 November 2019; Published: 11 November 2019

check for updates

\begin{abstract}
Diet plays an important role in the development of obesity and may contribute to dysregulated iron metabolism (DIM). A cross-sectional survey of 208 adults was conducted in Taipei Medical University Hospital (Taipei, Taiwan). A reduced-rank regression from 31 food groups was used for a dietary pattern analysis. DIM was defined as at least four of the following criteria: serum hepcidin (men $>200 \mathrm{ng} / \mathrm{mL}$ and women $>140 \mathrm{ng} / \mathrm{mL}$ ), hyperferritinemia (serum ferritin of $>300 \mathrm{ng} / \mathrm{mL}$ in men and $>200 \mathrm{ng} / \mathrm{mL}$ in women), central obesity, non-alcoholic fatty liver disease, and two or more abnormal metabolic profiles. Compared to non-DIM patients, DIM patients were associated with an altered body composition and had a 4.52-fold (95\% confidence interval (CI): (1.95-10.49); $p<0.001)$ greater risk of metabolic syndrome (MetS) after adjusting for covariates. A DIM-associated dietary pattern (high intake of deep-fried food, processed meats, chicken, pork, eating out, coffee, and animal fat/skin but low intake of steamed/boiled/raw foods and dairy products) independently predicted central obesity (odds ratio (OR): 1.57; 95\% CI: 1.05-2.34; $p<0.05$ ) and MetS (OR: 1.89; 95\% CI: 1.07-3.35; $p<0.05$ ). Individuals with the highest DIM pattern scores (tertile 3 ) had a higher visceral fat mass (\%) ( $\beta=0.232 ; 95 \%$ CI: $0.011-0.453 ; p<0.05)$ but lower skeletal muscle mass $(\%)$ ( $\beta=-1.208$; 95\% CI: $-2.177-0.239 ; p<0.05)$ compared to those with the lowest DIM pattern scores (tertile 1). In conclusion, a high score for the identified DIM-associated dietary pattern was associated with an unhealthier body composition and a higher risk of MetS.
\end{abstract}


Keywords: central obesity; dysregulated iron metabolism; hepcidin; ferritin; dietary pattern; visceral fat; skeletal muscle mass; metabolic syndrome

\section{Introduction}

Obesity is a major public health problem which has substantially increased worldwide in recent decades. In Taiwan, the prevalence of being overweight and obese among adult is $43.4 \%$ according to the 2013-2014 Nutrition and Health Survey [1]. A recent meta-analysis showed that iron homeostasis is affected by obesity [2]. Dysregulated iron metabolism (DIM) was first observed by Moirand and Deugnier in 1997, who described a new clinical symptom of hepatic iron overload in patients without hemochromatosis (HFE) [3]. Recently, DIM was indicated in a wide range of diseases including cancer [4], polycythemia vera [5], and skeletal muscle and nerve degeneration [6]. Dysmetabolic iron overload syndrome (DIOS), a more-precise clinical definition for hepatic iron accumulation, is a condition characterized by mild liver iron overload, elevated serum ferritin and hepcidin, but normal or low serum iron/transferrin saturation (TS) together with the presence of metabolic disorders such as obesity, insulin resistance (IR), hypertension, and dyslipidemia [7,8]. Deugnier and colleagues estimated that $34.5-51.5 \%$ of patients with non-alcoholic fatty liver disease (NAFLD) had DIOS [8].

Obese people are reported to have excessive visceral fat accumulation (also known as abdominal or central obesity) and have a higher risk of metabolic syndrome (MetS) [5]. Several population-based surveys showed a strong link between elevated serum ferritin levels and a risk of MetS [3,9], and this observation was confirmed in a systemic review and meta-analysis [10]. Elevated serum ferritin (also known as hyperferritinemia) not only reflects hepatic iron levels [11] but also indicates an increased level of adipose tissue inflammation [12] or visceral fat mass [13], and impaired skeletal muscle mass [6]. Hence, DIM may directly contribute to the pathology of obesity-related metabolic complications through alterations in the body composition.

Elevated serum hepcidin concentrations are considered to be the underlying cause of DIM $[7,8]$. Hepcidin is a peptide hormone mainly released by hepatocytes and which acts as a negative iron regulator [14]. Generally, hepcidin maintains systemic iron homeostasis by controlling the duodenal absorption of iron and the release of stored iron into plasma [15]. Hepcidin is stimulated by multiple factors, including the body's iron status (plasma iron, hypoxia, and anemia), erythropoiesis activity, hormones (e.g., estrogen and leptin), and inflammation $[7,14,16]$. The main role of hepcidin is to regulate the only known mammalian iron exporter, ferroportin [14]. Elevated hepcidin expression leads to ferroportin degradation, resulting in elevation of iron concentrations within cells and low circulating iron levels [17]. On the other hand, a lack of hepcidin expression results in elevation of ferroportin expression which enhances the iron absorption rate [17].

Dietary factors play important roles in the development of obesity and may contribute to an iron imbalance. Studies showed that meat, fish, and poultry are positively associated with the serum iron status, while dairy products, bread, cereals/fiber, protein-based foods/soy protein, tea, and coffee are negatively correlated with iron status [18]. However, meat, particular red meat, is part of the Western diet (characterized by high intake of red meat, processed meats, refined carbohydrates, and fatty foods but low intake of vegetables, fruits, and whole grains), and Pacheho et al. reported that red meat consumption was positively correlated with ferritin levels and the risk of myocardial infarction (hazard ratio (HR) per $50 \mathrm{~g}$ of daily intake: 1.18 ; 95\% confidence interval (CI): (1.05-1.33) [19]. A study by Sabrina et al. reported that young adult women who consumed an iron: ferritin ratio dietary pattern (high intake of beef, lamb, dairy products, fruits, and whole grains but low intake of refined carbohydrates and deep- and stir-fried foods) were associated with a healthy body composition and a $90 \%$ reduced risk of fatty liver progression (odds ratio (OR): $0.10 ; 95 \%$ confidence interval (CI): $0.02-0.47 ; p<0.001)[13]$. 
Currently, the role of diet in the development of DIM remains unclear. A reduced-rank regression (RRR) analysis, a data-driven (exploratory) method to characterize major patterns of food intake, is regarded as an approach to dissect the link between diet and chronic diseases [20]. The broad aim of this study was to investigate dietary patterns that are associated with DIM and their predictive effects on the body composition and MetS in 208 Taiwanese adults. Specifically, we aimed to: (1) identify dietary pattern scores associated with concentrations of serum biomarkers related to DIM using an RRR analysis and (2) to test whether the dietary pattern obtained was prospectively associated with the body composition, central obesity, and MetS.

\section{Materials and Methods}

\subsection{Participants}

This study was conducted in accordance with the Declaration of Helsinki (1964) and its later amendments (2013). The study protocol was reviewed and approved by the Taipei Medical University Institutional Ethical Review Committee (TMU-JIRB 201502018). Written informed consent was signed by all eligible subjects. Briefly, this was a cross-sectional study, and the sampling method was a non-probability sampling. Taiwanese adults $(n=230)$ aged $20-65$ years were enrolled through the recruitment process at the Division of Gastroenterology and Hepatobiliary Disease, Department of Internal Medicine, Taipei Medical University Hospital (Taipei, Taiwan), from July 2015 to June 2016. The sample size was calculated based on studies by Deugnier et al. [8] and Dongiovanni et al. [21]. With a two-sided significance level of $95 \%$ and a power of $80 \%$, the calculated sample size was 32 per group. Twenty-two subjects were excluded from the study because 12 persons had one of the following criteria: (1) a hepatitis virus (HV) infection history (e.g., HVA, HVB, or HVC) or liver malignancy; (2) thalassemia, polycystic ovary syndrome, or drug-induced hepatitis history; (3) excessive alcohol intake (alcohol intake $>20 \mathrm{~g} /$ week for women or $>30 \mathrm{~g} /$ week for men); (4) pregnancy or breastfeeding; and (5) use of hormone-replacement therapy or iron supplementation. One person declined to participate, and nine persons did not have blood data. Only 208 subjects ( 105 men and 103 women) were eligible and with no missing data were included in the final analysis. The reporting of this study conformed to the Strengthening the Reporting of Observational Studies in Epidemiology (STROBE) and Enhancing the Quality and Transparency of Health Research (EQUATOR) guidelines (Supplementary Table S1) [22,23].

\subsection{Definitions}

Currently, there is still no consensus for DIM diagnostic criteria. However, there are some clinical features that are associated with DIM, such as alterations in iron and metabolic profiles. Therefore, we defined DIM as the presence of at least four of the following criteria: (1) elevated serum hepcidin ( $>200 \mathrm{ng} / \mathrm{mL}$ in men and $>140 \mathrm{ng} / \mathrm{mL}$ in women); (2) hyperferritinemia (serum ferritin (SF) $>300 \mathrm{ng} / \mathrm{mL}$ in men and $>200 \mathrm{ng} / \mathrm{mL}$ in women) [14]; (3) central obesity (waist circumference (WC) of $\geq 90 \mathrm{~cm}$ in men and $\geq 80 \mathrm{~cm}$ in women) [24,25]; (4) NAFLD; and (5) the presence of at least two abnormal metabolic profiles (1) a fasting triglyceride (TG) level of $\geq 150 \mathrm{mg} / \mathrm{dL}$; (2) a high-density lipoprotein cholesterol (HDL-C) level of $<40 \mathrm{mg} / \mathrm{dL}$ in men and $<50 \mathrm{mg} / \mathrm{dL}$ in women; (3) systolic blood pressure (BP) of $\geq 130 \mathrm{mmHg}$ or diastolic BP of $\geq 85 \mathrm{mmHg}$; and (4) a fasting plasma glucose (FPG) concentration of $\geq 100 \mathrm{mg} / \mathrm{dL}$. Abdominal ultrasound was used to screen individuals with NAFLD based on the following criteria: parenchymal brightness, liver-to-kidney contrast, deep-beam attenuation, bright vessel walls, and gallbladder wall definition. An abnormal serum alanine aminotransferase (ALT) level was defined as $>40 \mathrm{U} / \mathrm{L}$. Anemia was defined as hemoglobin $(\mathrm{Hb})$ of $<13 \mathrm{~g} / \mathrm{dL}$ in men and $<12 \mathrm{~g} / \mathrm{dL}$ in women. Iron-deficiency anemia (IDA) was defined as two or more indicators of the iron status being abnormal: (1) $\mathrm{Hb}$ of $<13 \mathrm{~g} / \mathrm{dL}$ in men and $<12 \mathrm{~g} / \mathrm{dL}$ in women; (2) SF of $<12 \mu \mathrm{g} / \mathrm{L}$; and (3) TS of $<15 \%[14,26]$. MetS was determined based on the modified National Cholesterol Education Program Adult Treatment Panel III for the Asia Pacific [27,28]: with three of more of the following criteria: fasting TG of $\geq 150 \mathrm{mg} / \mathrm{dL}$; HDL-C of $<40 \mathrm{mg} / \mathrm{dL}$ in men and $<50 \mathrm{mg} / \mathrm{dL}$ in women; systolic BP of 
$\geq 130 \mathrm{mmHg}$ or diastolic BP of $\geq 85 \mathrm{mmHg}$; and an FPG of $\geq 100 \mathrm{mg} / \mathrm{dL}$ [14]. Dyslipidemia was defined when a subject had at least one of the following criteria: (1) TG level of $\geq 200 \mathrm{mg} / \mathrm{dL}$; (2) total cholesterol (TC) level of $\geq 240 \mathrm{mg} / \mathrm{dL}$; (3) HDL-C level of $<35 \mathrm{mg} / \mathrm{dL}$; (4) low-density lipoprotein (LDL)-C level of $\geq 160 \mathrm{mg} / \mathrm{dL}$; (5) TC/HDL-C ratio of $\geq 5$; and (6) use of lipid-lowering medicine [14]. Diabetes mellitus (DM) was defined when a subject had at least one of the following criteria: (1) an FPG concentration or $\geq 126 \mathrm{mg} / \mathrm{dL}$ and (2) glycated $\mathrm{Hb}$ (HbA1C) of $\geq 6.5 \%$ [14].

\subsection{Questionnaires}

A basic questionnaire was used to obtain general information about the subjects, including age, sex, nationality, anthropometric data, health status/medical history, medication use, and alcohol consumption. A modified self-reporting food frequency questionnaire (FFQ) based on the Chinese version of the FFQ for the Taiwanese population [29] was used to evaluate: (1) the usual weekly intake frequency of 66 food items which were grouped into 31 food groups according to similarities; (2) five types of commonly used cooking methods; and (3) the frequency of eating out. The self-report FFQ consisted with eight scales: (1) $\leq 1$ times/week; (2) 2 or 3 times/week; (3) 4 or 5 times/week; (4) 6 or 7 times/week; (5) 8 10 times/week; (6) 11 13 times/week; (7) 14 16 times/week; and (8) $\geq 17$ times/week. To reduce the information bias, the content validity of the modified FFQ was assessed by three nutrition experts. The internal consistency/reliability of the modified FFQ was high with Cronbach's alpha coefficient of 0.85 .

\subsection{Anthropometric Measurements}

Body weight (BW), height, and WC were measured for each subject by the investigator. The body-mass index (BMI) was determined as the BW divided by body height squared $\left(\mathrm{kg} / \mathrm{m}^{2}\right)$. WC was measured at the midpoint between the bottom rib and the top of the hip bone (iliac crest). A direct segmental multi-frequency bioelectrical impedance analysis (DSM-BIA) was conducted using a DSM-BIA meter X-SCAN Plus-II analyzer (Jawon, Seoul, Korea).

\subsection{Laboratory Measurements}

Fasting blood was collected from overnight-fasted participants. Briefly, blood tests included a complete blood cell count, glucose and lipid profiles, liver injury and oxidative stress biomarkers, and serum iron biomarkers. Fasting plasma insulin was assessed by a radioimmunoassay (Millipore, Billerica, MA, USA). FPG was quantitated by the glucose oxidase method. Serum TC, TG, and HDL-C were determined using reagents from Beckman Laboratories and quantitated by an autoanalyzer (Beckman Coulter Unicel DxC 800, Taipei, Taiwan). The LDL-C level was calculated using the Friedewald formula (LDL-C $=\mathrm{TC}-(\mathrm{HDL}-\mathrm{C}+\mathrm{TG} / 5) \mathrm{mg} / \mathrm{dL}$ ). Hb was measured by the direct cyanometahemoglobin method, which is the gold standard (Merckotest, Merck, Taipei, Taiwan). A ferrozine-based colorimetric method was used to quantitate serum iron. SF was assessed by an electrochemiluminescence immunoassay and was quantitated with a Roche Modular P800 analyzer (Roche; Taipei, Taiwan). Serum hepcidin was assessed by an enzyme-linked immunosorbent assay (ELISA) according to the manufacturer's instructions (DRG International; Springfield Township, NJ, USA). Percentage TS (\%TS) was calculated as (serum iron/total iron-binding capacity (TIBC)) $\times 100 \%$. $\mathrm{HbA1C}$ was assessed by an affinity high-performance liquid chromatography (HPLC) method. Serum ALT was measured by a colorimetric method with the Beckman DxC 800 (Beckman Coulter). The serum malondialdehyde (MDA) concentration, a lipid peroxidation marker, was determined based on the reaction of MDA with thiobarbituric acid to form thiobarbituric acid-reactive species. Serum nitric oxide (NO) was assessed with the Griess reagent (Sigma-Aldrich, Taipei, Taiwan).

\subsection{Statistical Analysis}

Statistical analyses were performed using the IBM SPSS statistics software vers. 20 (IBM, Armonk, NY, USA) and SAS 9.4 (SAS Institute, Cary, NC, USA). Categorical data are presented as the number 
(percentage (\%)), and continuous data are presented as the mean \pm standard deviation (SD). A general linear model was used to analyze the $p$ for trend. Multiple comparison analysis testing in a one-way analysis of variance (ANOVA) (with the Bonferroni post-hoc correction) was used to compare multiple groups. A chi-squared test was used to compare categorical variables. An RRR analysis was applied to derive DIM-associated dietary patterns. The RRR was originally described by Hoffmann and colleagues to delineate the relationship between diet and chronic diseases [20]. This study used 31 food groups from the FFQ as predictor variables. Serum hepcidin, ferritin, ALT, and HDL-C were selected as response variables based on their strong correlations with DIM. For dietary pattern scores, only food groups that had factor loadings greater than or equal to positive/negative $0.20(\geq|0.20|)$ were selected. Factor loading indicates the correlation between a food group and the RRR-derived dietary pattern. A dietary pattern score was calculated for each participant by deriving a score that represents the sum of food intake variables weighted by factor loadings to reflect their intake conformity with the DIM-associated dietary pattern. A multivariate logistic regression was performed to identify risk factors associated with DIM and determine the role of the dietary pattern score in predicting the risk of DIM and MetS. A multivariate linear regression was used to evaluate the relationship between DIM-associated dietary pattern scores and body composition. The direct acyclic graph in Figure 1 explains the conceptual framework of the RRR. A $p$ value of $\leq 0.05$ was considered statistically significant for all analyses. The figure was created using GraphPad Prism vers. 5 (GraphPad Software, La Jolla, CA, USA).

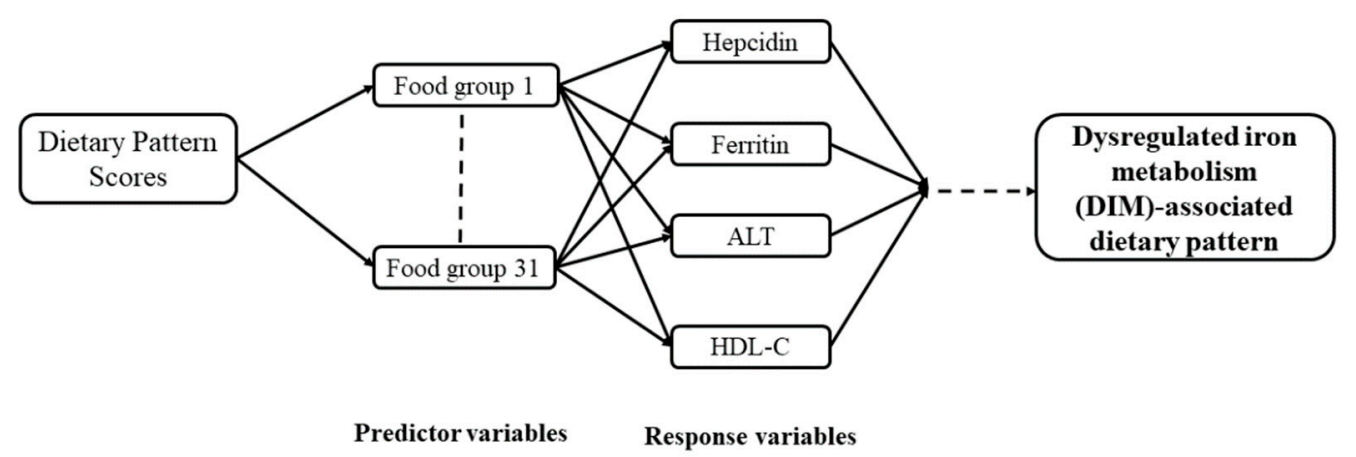

Figure 1. Direct acyclic graph of the reduced-rank regression (RRR) conceptual framework. ALT, alanine aminotransferase; HDL-C, high-density lipoprotein cholesterol.

\section{Results}

\subsection{Baseline Characteristics of the Study Population According to Dysregulated Iron Metabolism (DIM)}

Table 1 shows baseline characteristics of study participants according to their DIM status. Mean ages were $40.55 \pm 1.01$ years for non-DIM subjects and $47.15 \pm 1.58$ years for DIM patients $(p=0.001)$. Prevalence rates of DIM and MetS were $22.6 \%$ and $24.5 \%$, respectively. In general, DIM patients were older and heavier, and had a higher incidence of metabolic diseases (e.g., NAFLD, MetS, central obesity, $\mathrm{DM}$, dyslipidemia, and hypertension), higher levels of circulating iron (e.g., $\mathrm{Hb}$, serum hepcidin, and SF), liver injury and oxidative stress biomarkers (NO, MDA, and ALT), blood lipids (high TG and LDL-C but low HDL-C), and glucose biomarkers (FPG and HbA1C) compared to non-DIM subjects (all $p<0.05$; Table 1). In addition, anthropometric measurements showed that DIM patients were associated with a higher BMI, WC, body fat mass, and visceral fat mass but lower skeletal muscle mass compared to non-DIM patients (all $p<0.001$; Table 1). 
Table 1. Baseline characteristics of the study population according to the dysregulated iron metabolism (DIM) status $(N=208)$.

\begin{tabular}{|c|c|c|c|}
\hline \multirow{2}{*}{ Characteristic } & \multicolumn{2}{|c|}{ Study Population $(N=208)$} & \multirow{2}{*}{$p$ Value * } \\
\hline & $\operatorname{DIM}(-)(n=161)$ & $\operatorname{DIM}(+)(n=47)$ & \\
\hline Age (years) & $40.55 \pm 12.76$ & $47.15 \pm 10.82$ & 0.001 \\
\hline \multicolumn{4}{|l|}{ Gender $(n, \%)$} \\
\hline Male & $81(50.3)$ & $24(51.1)$ & 0.928 \\
\hline Female & $80(49.7)$ & $23(48.9)$ & \\
\hline $\operatorname{NAFLD}(n, \%)$ & $124(77.0)$ & $46(97.9)$ & 0.001 \\
\hline $\operatorname{MetS}(n, \%)$ & $22(13.7)$ & $29(61.7)$ & $<0.001$ \\
\hline Central obesity $(n, \%)$ & $54(33.5)$ & $45(95.7)$ & $<0.001$ \\
\hline Diabetes mellitus $(n, \%)$ & $15(9.3)$ & $10(21.3)$ & 0.027 \\
\hline Dyslipidemia $(n, \%)$ & $32(19.9)$ & $28(59.6)$ & $<0.001$ \\
\hline Hypertension $(n, \%)$ & $69(42.9)$ & $35(74.5)$ & $<0.001$ \\
\hline Abnormal ALT $(n, \%)$ & 19 (11.8) & $23(48.9)$ & $<0.001$ \\
\hline \multicolumn{4}{|c|}{ Anthropometry } \\
\hline BMI $\left(\mathrm{kg} / \mathrm{m}^{2}\right)$ & $23.41 \pm 4.37$ & $28.59 \pm 5.77$ & $<0.001$ \\
\hline Waist circumference $(\mathrm{cm})$ & $83.04 \pm 11.80$ & $96.86 \pm 12.88$ & $<0.001$ \\
\hline Male & $87.98 \pm 9.32$ & $101.63 \pm 10.26$ & $<0.001$ \\
\hline Female & $78.04 \pm 11.98$ & $91.88 \pm 13.65$ & $<0.001$ \\
\hline Body fat mass (\%) & $26.47 \pm 5.90$ & $32.38 \pm 5.53$ & $<0.001$ \\
\hline Skeletal muscle mass (\%) & $67.79 \pm 5.83$ & $61.93 \pm 5.48$ & $<0.001$ \\
\hline Visceral fat mass (\%) & $3.37 \pm 1.18$ & $4.85 \pm 1.23$ & $<0.001$ \\
\hline \multicolumn{4}{|c|}{ Glucose biomarkers } \\
\hline $\begin{array}{l}\text { Fasting blood glucose } \\
(\mathrm{mg} / \mathrm{dL})\end{array}$ & $89.83 \pm 16.35$ & $97.36 \pm 24.81$ & 0.045 \\
\hline Insulin (mIU/mL) & $9.62 \pm 5.43$ & $9.11 \pm 6.08$ & 0.327 \\
\hline $\mathrm{HbA} 1 \mathrm{C}(\%)$ & $5.72 \pm 0.83$ & $6.16 \pm 1.31$ & 0.001 \\
\hline \multicolumn{4}{|c|}{ Lipid biomarkers } \\
\hline Total cholesterol (mg/dL) & $197.45 \pm 37.59$ & $206.49 \pm 38.13$ & 0.052 \\
\hline TGs (mg/dL) & $102.05 \pm 67.34$ & $174.23 \pm 67.47$ & $<0.001$ \\
\hline HDL-C (mg/dL) & $59.00 \pm 16.25$ & $48.80 \pm 11.81$ & $<0.001$ \\
\hline LDL-C (mg/dL) & $115.99 \pm 32.15$ & $127.85 \pm 31.96$ & 0.012 \\
\hline \multicolumn{4}{|c|}{ Iron-related biomarkers } \\
\hline $\mathrm{Hb}(\mathrm{gm} / \mathrm{dL})$ & $14.55 \pm 2.41$ & $15.66 \pm 3.12$ & 0.003 \\
\hline Iron $(\mu \mathrm{g} / \mathrm{dL})$ & $102.97 \pm 38.63$ & $107.13 \pm 34.14$ & 0.632 \\
\hline Hepcidin (ng/mL) & $118.61 \pm 93.54$ & $259.77 \pm 67.59$ & $<0.001$ \\
\hline Ferritin (ng/mL) & $117.16 \pm 121.84$ & $263.64 \pm 169.26$ & $<0.001$ \\
\hline TS (\%) & $29.69 \pm 12.62$ & $30.33 \pm 10.31$ & 0.765 \\
\hline Elevated hepcidin $(n, \%)$ & $38(23.6)$ & $46(97.9)$ & $<0.001$ \\
\hline Anemia $(n, \%)$ & $13(8.1)$ & $2(4.3)$ & 0.373 \\
\hline $\begin{array}{l}\text { Iron-deficiency anemia } \\
\qquad(n, \%)\end{array}$ & $14(8.7)$ & $0(0.0)$ & 0.036 \\
\hline Hyperferritinemia $(n, \%)$ & $8(5.0)$ & $26(55.3)$ & $<0.001$ \\
\hline \multicolumn{4}{|c|}{ Liver injury and oxidative stress biomarkers } \\
\hline Nitrite oxide $(\mu \mathrm{M})$ & $44.44 \pm 28.01$ & $61.67 \pm 24.33$ & $<0.001$ \\
\hline $\operatorname{ALT}(\mathrm{U} / \mathrm{L})$ & $26.14 \pm 17.88$ & $54.96 \pm 37.98$ & $<0.001$ \\
\hline $\operatorname{MDA}(\mu \mathrm{M})$ & $40.13 \pm 27.54$ & $47.89 \pm 21.23$ & 0.005 \\
\hline
\end{tabular}

DIM was defined by the presence of at least four of the following five criteria: elevated serum hepcidin $(>200 \mathrm{ng} / \mathrm{mL}$ in males and $>140 \mathrm{ng} / \mathrm{mL}$ in females), hyperferritinemia (serum ferritin $>300 \mathrm{ng} / \mathrm{mL}$ in males and $>200 \mathrm{ng} / \mathrm{mL}$ in females), central obesity ( $>90 \mathrm{~cm}$ in males and $>80 \mathrm{~cm}$ in females), non-alcoholic fatty liver disease (NAFLD), and at least two abnormal metabolic profiles. Continuous data are presented as the mean \pm standard deviation; categorical data are presented as numbers (percentages); * The $p$ value was analyzed using the Mann-Whitney test for continuous variables and chi-squared test for categorical variables. MetS, metabolic syndrome; BMI, body-mass index; HbA1C, glycated hemoglobin; TGs, triglycerides; HDL-C, high-density lipoprotein-cholesterol; LDL-C, low-density lipoprotein-cholesterol; $\mathrm{Hb}$, hemoglobin; TS, transferrin saturation; ALT, alanine aminotransferase; MDA, malondialdehyde. 


\subsection{DIM and Risk of Altered Body Composition and Metabolic Syndrome (MetS)}

We next performed a multivariate linear and logistic regression analysis to evaluate relationships among DIM, body composition, and MetS. Figure 2A shows that compared to non-DIM individuals, individuals with DIM were associated with increased \% visceral fat mass $(\beta=1.355(0.961-1.749)$, $p<0.001)$ and decreased \% skeletal muscle mass $(\beta=-5.417(-7.094--3.740), p<0.001)$ after adjusting for age and sex. A multivariate logistic regression analysis showed that compared to non-DIM subjects, DIM patients had 4.52 (OR: 4.52; 95\% CI: 1.95-10.49; $p<0.001$ ) higher risks of developing MetS after adjusting for age, gender, and BMI (Figure 2B).

(A)

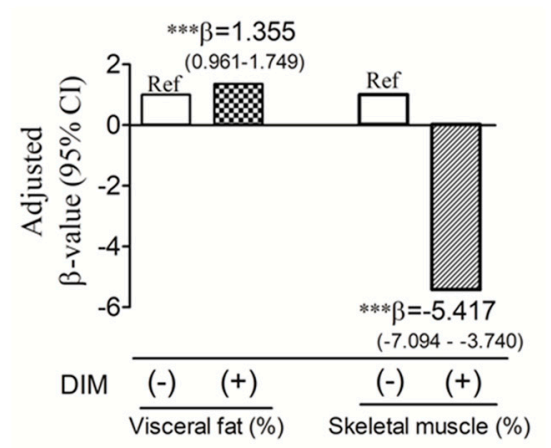

(B)

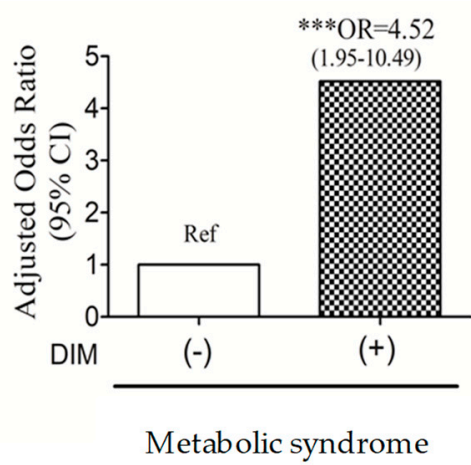

Figure 2. Adjusted multivariate linear and logistic regression and $95 \%$ confidence intervals of dysregulated iron metabolism (DIM) in terms of visceral fat mass (\%) and skeletal muscle mass (\%) (A) and metabolic syndrome (B). (A) the Beta coefficient ( $\beta$-value) was adjusted by age and sex, and (B) odds ratio (OR) was adjusted by age, sex, and body-mass index (BMI) $(N=208) .{ }^{* * *} p \leq 0.001$.

\subsection{DIM-Associated Dietary Pattern Scores by the Reduced-Rank Regression (RRR)}

Currently, dietary risk factors associated with DIM remain undefined. We applied an RRR to derive a predictive dietary pattern for DIM. Response variables for the RRR were selected based on relationships between independent variables and DIM. A multivariate logistic regression analysis revealed that serum hepcidin $(\mathrm{OR}=1.018(1.010-1.025) ; p \leq 0.001)$, SF $(\mathrm{OR}=1.007$ (1.002-1.012); $p=0.005)$, and ALT (OR $=1.029(1.004-1.055) ; p=0.021)$ were positively correlated with DIM, while HDL-C (OR $=0.929(0.865-0.999) ; p=0.046)$ was negatively correlated with DIM (Table 2$)$.

We next investigated relationships of selected response variables (hepcidin, ferritin, ALT, and HDL-C) with the visceral fat mass and skeletal muscle mass. The multivariate linear regression analysis found positive trends between the percent visceral fat mass and hepcidin $(\beta=0.001 ; 95 \% \mathrm{CI}$ : 0.0001-0.002; $p<0.05)$, and ALT ( $\beta=0.004 ; 95 \%$ CI: 0.001-0.007; $p<0.05)$ after adjusting for age, sex, and the BMI (Table 3, model 3 ). In contrast, the skeletal muscle mass was positively correlated with HDL-C $(\beta=0.032 ; 95 \%$ CI: 0.004-0.060; $p<0.05)$ and negatively correlated with hepcidin $(\beta=-0.004$; 95\% CI: $-0.008--0.0001 ; p<0.05$ and ALT $(\beta=-0.020 ; 95 \%$ CI: $-0.035-0.004 ; p<0.05)$ after adjusting for age, sex, and the BMI (Table 3, model 3). 
Table 2. Multivariate logistic regression of risk factors associated with dysregulated iron metabolism $(N=208)$.

\begin{tabular}{|c|c|c|c|c|c|c|c|c|}
\hline \multirow{3}{*}{$\begin{array}{l}\text { Variable } \\
\text { Age (years) }\end{array}$} & \multicolumn{4}{|c|}{ Univariate } & \multicolumn{4}{|c|}{ Multivariate * } \\
\hline & \multirow{2}{*}{$\begin{array}{c}\text { OR } \\
1.044\end{array}$} & \multirow{2}{*}{$\begin{array}{l}\mathbf{9 5} \% \mathrm{CI} \\
1.02\end{array}$} & \multicolumn{2}{|c|}{$p$ Value } & \multirow{2}{*}{$\begin{array}{c}\text { OR } \\
1.036\end{array}$} & \multicolumn{2}{|c|}{$95 \%$ CI } & \multirow{2}{*}{$\begin{array}{c}p \text { Value } \\
0.235\end{array}$} \\
\hline & & & 1.07 & 0.002 & & 0.977 & 1.099 & \\
\hline \multicolumn{9}{|l|}{$\operatorname{Sex}(n, \%)$} \\
\hline Female & Ref & & & & & & & \\
\hline Male & 1.031 & 0.54 & 1.97 & 0.928 & & & & \\
\hline $\operatorname{BMI}\left(\mathrm{kg} / \mathrm{m}^{2}\right)$ & 1.224 & 1.13 & 1.32 & $<0.001$ & & & & \\
\hline Hemoglobin (g/dL) & 1.159 & 1.03 & 1.31 & 0.014 & & & & \\
\hline $\operatorname{Iron}(\mu \mathrm{g} / \mathrm{dL})$ & 1.003 & 0.99 & 1.01 & 0.504 & & & & \\
\hline Hepcidin (ng/mL) & 1.02 & 1.01 & 1.02 & $<0.001$ & 1.018 & 1.010 & 1.025 & $<0.001$ \\
\hline Ferritin (ng/mL) & 1.01 & 1.00 & 1.01 & $<0.001$ & 1.007 & 1.002 & 1.012 & 0.005 \\
\hline Transferrin saturation (\%) & 1.01 & 0.98 & 1.03 & 0.753 & & & & \\
\hline ALT (U/L) & 1.04 & 1.03 & 1.06 & $<0.001$ & 1.029 & 1.004 & 1.055 & 0.021 \\
\hline $\operatorname{MDA}(\mu \mathrm{M})$ & 1.01 & 0.998 & 1.02 & 0.115 & & & & \\
\hline Total cholesterol (mg/dL) & 1.01 & 0.998 & 1.02 & 0.151 & & & & \\
\hline Triglycerides (mg/dL) & 1.01 & 1.01 & 1.02 & $<0.001$ & 1.003 & 0.993 & 1.012 & 0.582 \\
\hline LDL-C (mg/dL) & 1.0 & 1.00 & 1.02 & 0.029 & & & & \\
\hline HDL-C (mg/dL) & 0.944 & 0.916 & 0.97 & $<0.001$ & 0.929 & 0.865 & 0.999 & 0.046 \\
\hline Fasting blood glucose (mg/dL) & 1.02 & 1.00 & 1.03 & 0.023 & & & & \\
\hline Insulin (mIU/mL) & 0.983 & 0.906 & 1.07 & 0.679 & & & & \\
\hline $\operatorname{HbA} 1 \mathrm{C}(\%)$ & 1.48 & 1.07 & 2.05 & 0.018 & 0.689 & 0.335 & 1.41 & 0.309 \\
\hline
\end{tabular}

* The multivariate was adjusted for age, gender, body-mass index (BMI), hepcidin, ferritin, alanine transaminase (ALT), triglycerides, HbA1c, and high-density lipoprotein cholesterol (HDL-C). DIM was defined by the presence of at least four of the following five criteria: elevated serum hepcidin $(>200 \mathrm{ng} / \mathrm{mL}$ in males and $>140 \mathrm{ng} / \mathrm{mL}$ in females), elevated serum ferritin ( $>300 \mathrm{ng} / \mathrm{mL}$ in males and $>200 \mathrm{ng} / \mathrm{mL}$ in females), central obesity ( $>90 \mathrm{~cm}$ in males and $>80 \mathrm{~cm}$ in females), non-alcoholic fatty liver disease, and at least two abnormal metabolic profiles. Abbreviations: CI, confidence interval; MDA, malondialdehyde; LDL-C, low-density lipoprotein cholesterol.

Table 3. Associations of selected response variables with the visceral fat mass and skeletal muscle mass $(N=208)$.

\begin{tabular}{|c|c|c|c|c|c|c|}
\hline & \multicolumn{6}{|c|}{ Visceral Fat Mass (\%) } \\
\hline & \multicolumn{2}{|l|}{ \# Model 1} & \multicolumn{2}{|l|}{ * Model 2} & \multicolumn{2}{|l|}{ \& Model 3} \\
\hline & $\beta(95 \% \mathrm{CI})$ & $p$ Value & $\beta(95 \% \mathrm{CI})$ & $p$ Value & $\beta(95 \% \mathrm{CI})$ & $p$ Value \\
\hline Hepcidin (ng/mL) & $0.003(0.002-0.005)$ & $<0.001$ & $0.003(0.002-0.005)$ & $<0.001$ & $0.001(0.0001-0.002)$ & 0.025 \\
\hline Ferritin $(\mathrm{ng} / \mathrm{mL})$ & $0.002(0.0001-0.003)$ & 0.008 & $0.002(0.0001-0.003)$ & 0.030 & $1.187(-0.001-0.001)$ & 0.972 \\
\hline ALT (U/L) & $0.020(0.014-0.026)$ & $<0.001$ & $0.020(0.014-0.026)$ & $<0.001$ & $0.004(0.001-0.007)$ & 0.018 \\
\hline \multirow[t]{2}{*}{$\mathrm{HDL}(\mathrm{mg} / \mathrm{dL})$} & $-0.030(-0.041--0.020)$ & $<0.001$ & $-0.032(-0.044--0.021)$ & $<0.001$ & $-0.005(-0.011-0.001)$ & 0.109 \\
\hline & \multicolumn{6}{|c|}{ Skeletal muscle mass (\%) } \\
\hline Hepcidin (ng/mL) & $-0.003(-0.011-0.004)$ & 0.388 & $-0.014(-0.021--0.006)$ & $<0.001$ & $-0.004(-0.008--0.0001)$ & 0.033 \\
\hline Ferritin (ng/mL) & $0.005(-0.001-0.010)$ & 0.102 & $-0.006(-0.012-0.0001)$ & 0.039 & $-6.395(-0.003-0.003)$ & 0.968 \\
\hline ALT (U/L) & $-0.067(-0.097--0.038)$ & $<0.001$ & $-0.084(-0.110--0.059)$ & $<0.001$ & $-0.020(-0.035--0.004)$ & 0.012 \\
\hline $\mathrm{HDL}(\mathrm{mg} / \mathrm{dL})$ & $0.045(-0.007-0.097)$ & 0.087 & $0.142(0.094-0.191)$ & $<0.001$ & $0.032(0.004-0.060)$ & 0.027 \\
\hline
\end{tabular}

\# Model 1 was adjusted for age; * Model 2 was adjusted for age and gender; \& Model 3 was adjusted for age, gender, and body-mass index. ALT, alanine aminotransferase; HDL, high-density lipoprotein.

Table 4 shows percentages of food variations explained by the first dietary pattern scores and factor loadings of the food groups. The first dietary pattern derived by the RRR was characterized by high frequencies of deep-fried foods, processed meats, chicken, pork, eating out, coffee, and animal fat/skin but low intake frequencies of steamed/boiled/raw foods and dairy products (factor loadings $\geq$ $|0.20|$ ) (Table 4 ). This dietary pattern explained $64.73 \%$ of the total variation in the food groups. 
Table 4. Food groups that were strongly associated with the dysregulated iron metabolism-related dietary pattern scores identified using a reduced rank regression (RRR).

\begin{tabular}{ccc}
\hline Food Groups & Explained Variation (\%) & Factor Loading * \\
\hline Deep-fried foods & 15.87 & 0.41 \\
Processed meats & 11.08 & 0.34 \\
Chicken and pork & 7.88 & 0.29 \\
Eating out & 7.33 & 0.28 \\
Coffee & 4.85 & 0.23 \\
Animal fat/skin & 4.80 & 0.22 \\
Steamed/boiled/raw food & 7.57 & -0.28 \\
Dairy products & 5.36 & -0.24 \\
Total explained variation (\%): & 64.73 & \\
\hline
\end{tabular}

* Factor loadings show correlations between food groups and the first dietary pattern scores (correlation coefficient for the RRR-derived pattern $\geq|0.20|$ ).

\subsection{Relationships among DIM-Associated Dietary Pattern Scores, MetS, Central Obesity, and} Body Composition

Table 5 shows clinical characteristics and blood biochemical levels according to tertiles of dietary pattern scores. Participants with higher dietary pattern scores were more likely to be male, have central obesity (e.g., higher WC and visceral fat mass), have higher incidences of metabolic diseases (e.g., MetS or high TG but low HDL-C), and have higher levels of circulating iron (e.g., Hb, serum hepcidin, and ferritin) and the liver injury biomarker (ALT) (Table 5).

Table 5. Clinical characteristics and blood biochemical levels of the study population stratified by tertiles of dietary pattern scores.

\begin{tabular}{|c|c|c|c|c|c|}
\hline \multirow{2}{*}{ Variable } & \multicolumn{3}{|c|}{ Tertile of Dietary Pattern Scores $\$$} & \multirow{2}{*}{${ }^{*} p$ for Trend } & \multirow{2}{*}{${ }^{\#} p$ Value } \\
\hline & T1 $(N=69)$ & $\mathrm{T} 2(N=69)$ & T3 $(N=69)$ & & \\
\hline Age (years) & $41.32 \pm 13.80$ & $41.04 \pm 13.13$ & $43.81 \pm 10.86$ & 0.730 & 0.752 \\
\hline \multicolumn{6}{|l|}{$\operatorname{Sex}(n, \%)$} \\
\hline Male & $24(34.8)$ & $37(53.6)$ & $44(63.8)$ & 0.003 & 0.002 \\
\hline Female & $45(65.2)$ & $32(46.4)$ & $25(36.2)$ & & \\
\hline $\operatorname{NAFLD}(n, \%)$ & $56(81.2)$ & $55(79.7)$ & $58(84.1)$ & 0.798 & 1.000 \\
\hline Metabolic syndrome $(n, \%)$ & $10(14.5)$ & $18(26.1)$ & $22(31.9)$ & 0.052 & 0.051 \\
\hline Central obesity $(n, \%)$ & $26(37.7)$ & $32(46.4)$ & $40(58.0)$ & 0.057 & 0.051 \\
\hline Diabetes mellitus $(n, \%)$ & $7(10.1)$ & $11(15.9)$ & $7(10.1)$ & 0.483 & 1.000 \\
\hline Dyslipidemia $(n, \%)$ & $14(20.3)$ & $23(33.3)$ & $23(33.3)$ & 0.149 & 0.277 \\
\hline Hypertension $(n, \%)$ & $32(46.4)$ & $34(49.3)$ & $37(53.6)$ & 0.693 & 1.000 \\
\hline $\operatorname{DIM}(n, \%)$ & $9(13.0)$ & $13(18.8)$ & $25(36.2)$ & 0.003 & 0.003 \\
\hline Abnormal ALT $(n, \%)$ & $7(10.1)$ & $15(21.7)$ & $20(29.0)$ & 0.021 & 0.018 \\
\hline \multicolumn{6}{|c|}{ Anthropometry } \\
\hline BMI $\left(\mathrm{kg} / \mathrm{m}^{2}\right)$ & $23.13 \pm 5.13$ & $24.83 \pm 5.35$ & $25.60 \pm 4.62$ & 0.005 & 0.014 \\
\hline Waist circumference $(\mathrm{cm})$ & $81.69 \pm 13.13$ & $86.98 \pm 13.92$ & $89.41 \pm 11.52$ & 0.001 & 0.002 \\
\hline Male & $89.26 \pm 12.84$ & $91.72 \pm 11.54$ & $91.58 \pm 9.80$ & 0.415 & 1.000 \\
\hline Female & $77.65 \pm 11.50$ & $81.50 \pm 14.59$ & $85.59 \pm 13.43$ & 0.016 & 0.048 \\
\hline Body fat mass (\%) & $26.86 \pm 6.10$ & $27.97 \pm 6.12$ & $28.43 \pm 6.59$ & 0.142 & 0.427 \\
\hline Skeletal muscle mass (\%) & $67.40 \pm 6.02$ & $66.30 \pm 6.06$ & $65.85 \pm 6.53$ & 0.144 & 0.431 \\
\hline Visceral fat mass (\%) & $3.27 \pm 1.28$ & $3.75 \pm 1.33$ & $4.05 \pm 1.30$ & 0.001 & 0.002 \\
\hline
\end{tabular}


Table 5. Cont.

\begin{tabular}{|c|c|c|c|c|c|}
\hline \multirow{2}{*}{ Variable } & \multicolumn{3}{|c|}{ Tertile of Dietary Pattern Scores $\$$} & \multirow{2}{*}{${ }^{*} p$ for Trend } & \multirow{2}{*}{${ }^{\#} p$ Value } \\
\hline & $\mathrm{T} 1(N=69)$ & $\mathrm{T} 2(N=69)$ & T3 $(N=69)$ & & \\
\hline \multicolumn{6}{|c|}{ Glucose biomarkers } \\
\hline Fasting blood glucose (mg/dL) & $88.88 \pm 13.27$ & $92.96 \pm 17.93$ & $92.70 \pm 23.82$ & 0.236 & 0.708 \\
\hline Insulin $(\mathrm{mIU} / \mathrm{mL})$ & $10.02 \pm 6.24$ & $8.42 \pm 4.41$ & $9.84 \pm 5.92$ & 0.894 & 1.000 \\
\hline $\operatorname{HbA1C}(\%)$ & $5.67 \pm 0.69$ & $5.91 \pm 1.06$ & $5.88 \pm 1.12$ & 0.198 & 0.595 \\
\hline \multicolumn{6}{|c|}{ Lipid biomarkers } \\
\hline Total cholesterol (mg/dL) & $198.84 \pm 30.93$ & $199.25 \pm 40.46$ & $201.35 \pm 41.11$ & 0.697 & 1.000 \\
\hline Triglyceride (mg/dL) & $100.93 \pm 71.69$ & $121.74 \pm 76.02$ & $132.46 \pm 71.40$ & 0.012 & 0.036 \\
\hline HDL-C (mg/dL) & $61.73 \pm 16.02$ & $54.10 \pm 15.39$ & $54.50 \pm 15.34$ & 0.007 & 0.779 \\
\hline LDL-C (mg/dL) & $115.30 \pm 28.60$ & $119.81 \pm 34.02$ & $121.54 \pm 34.24$ & 0.260 & 0.021 \\
\hline \multicolumn{6}{|c|}{ Iron-related biomarkers } \\
\hline Hemoglobin (g/dL) & $14.31 \pm 2.57$ & $14.91 \pm 2.62$ & $15.24 \pm 2.60$ & 0.036 & 0.109 \\
\hline Iron $(\mu \mathrm{g} / \mathrm{dL})$ & $101.75 \pm 38.06$ & $107.06 \pm 39.20$ & $104.10 \pm 34.79$ & 0.713 & 1.000 \\
\hline Hepcidin (ng/mL) & $123.97 \pm 93.68$ & $150.94 \pm 115.39$ & $178.80 \pm 101.92$ & 0.002 & 0.007 \\
\hline Ferritin $(\mathrm{ng} / \mathrm{mL})$ & $103.59 \pm 130.04$ & $144.32 \pm 129.73$ & $204.96 \pm 162.65$ & $<0.001$ & $<0.001$ \\
\hline Transferrin saturation (\%) & $28.76 \pm 12.17$ & $31.07 \pm 12.88$ & $30.03 \pm 11.02$ & 0.536 & 1.000 \\
\hline Elevated serum hepcidin $(n, \%)$ & $12(17.4)$ & $21(30.4)$ & $31(44.9)$ & 0.010 & 0.007 \\
\hline Anemia $(n, \%)$ & $7(10.1)$ & $4(5.8)$ & $3(4.3)$ & 0.369 & 0.533 \\
\hline Iron-deficiency anemia $(n, \%)$ & $7(10.1)$ & $5(7.2)$ & $1(1.4)$ & 0.100 & 0.107 \\
\hline Hyperferritinemia $(n, \%)$ & $5(7.2)$ & $11(15.9)$ & $19(27.5)$ & 0.006 & 0.004 \\
\hline \multicolumn{6}{|c|}{ Liver injury and oxidative stress biomarkers } \\
\hline Nitrite oxide $(\mu \mathrm{M})$ & $41.97 \pm 21.76$ & $54.79 \pm 33.36$ & $48.90 \pm 26.90$ & 0.193 & 0.579 \\
\hline $\operatorname{ALT}(\mathrm{U} / \mathrm{L})$ & $25.77 \pm 18.71$ & $32.30 \pm 24.51$ & $40.14 \pm 33.35$ & 0.001 & 0.004 \\
\hline MDA $(\mu \mathrm{M})$ & $43.41 \pm 30.40$ & $38.21 \pm 19.32$ & $44.69 \pm 28.12$ & 0.798 & 1.000 \\
\hline
\end{tabular}

Continuous data are presented as the mean \pm standard deviation; categorical data are presented as numbers (percentages). ${ }^{*} p$ for trend was analyzed by a general linear model for continuous variables and chi-squared test for categorical variables. \# The $p$ value was analyzed by a one-way analysis of variance (ANOVA) with the post-hoc Bonferroni correction. ${ }^{\$}$ Tertile of dietary pattern scores: $\mathrm{T} 1 \leq-0.303 ;-0.302 \leq \mathrm{T} 2 \leq 0.306$; T3 $>0.306$. NAFLD, non-alcoholic fatty liver disease; MetS, metabolic syndrome; DIM, dysregulated iron metabolism; BMI, body-mass index; HDL-C, high-density lipoprotein cholesterol; LDL-C, low-density lipoprotein cholesterol; Hb, hemoglobin; TS, transferrin saturation; ALT, alanine aminotransferase; MDA, malondialdehyde.

The multivariate logistic regression analysis showed that DIM-associated dietary pattern scores independently predicted DIM (OR: 3.34; 95\% CI: 1.73-6.44; $p<0.001$ ), MetS (OR: 1.89; 95\% CI: 1.07-3.35; $p<0.05$ ), and central obesity (OR: 1.57; 95\% CI: 1.05-2.34; $p<0.05$ ) after adjusting for age, sex, and BMI (Figure 3A). We next investigated relationships between DIM-associated dietary pattern scores and body composition. A multivariate linear regression analysis adjusted for age, sex, and BMI showed that individuals with the highest DIM pattern scores (tertile 3$)$ had increased visceral fat mass $(\%)(\beta$ $=0.232 ; 95 \%$ CI: 0.011-0.453; $p<0.05)$ but decreased skeletal muscle mass $(\%)(\beta=-1.208 ; 95 \%$ CI: $-2.177--0.239 ; p<0.05)$ compared to those with the lowest scores (tertile 1) (Figure 3). 


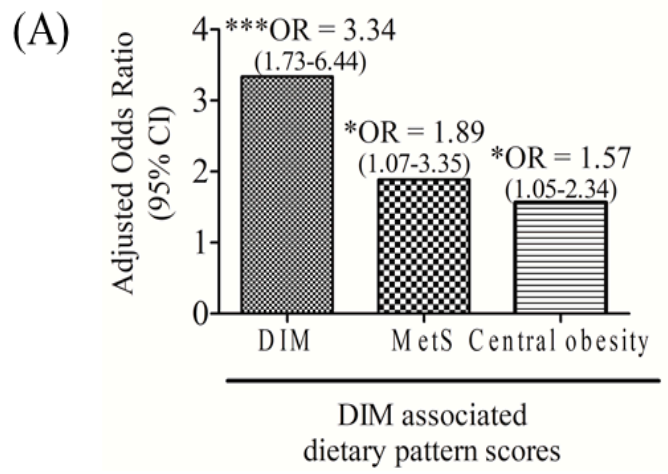

(B)

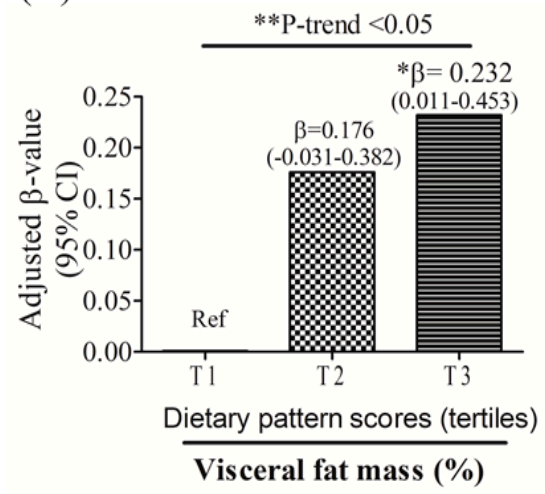

(C)

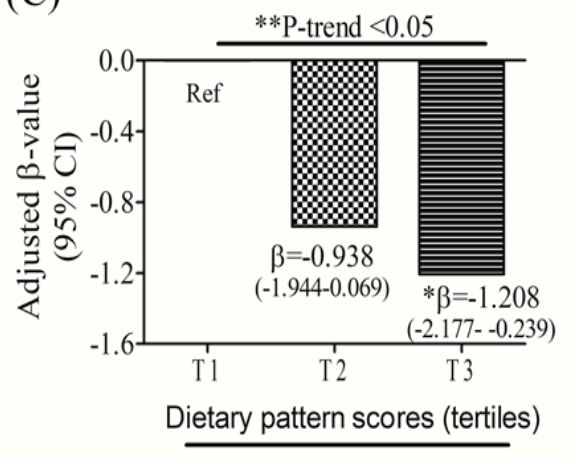

Skeletal muscle mass (\%)

Figure 3. Multivariate logistic analysis adjusted for age, gender, and body-mass index and linear regression and $95 \%$ confidence intervals (CIs) of dysregulated iron metabolism (DIM)-associated dietary pattern scores for predicting DIM (A), metabolic syndrome (MetS) (A), central obesity (A), visceral fat mass (\%) (B), and skeletal muscle mass (\%) (C). ${ }^{*} p<0.05 ;{ }^{* *} p<0.01 ;{ }^{* * *} p \leq 0.001$.

\section{Discussion}

To our knowledge, this is the first study to investigate dietary risk factors associated with DIM. Our results suggest that individuals who tend to eat out and that have a preference for a Western diet (indicated by high intake frequencies of meat, coffee, and fatty and deep-fried foods and low intake of steamed/boiled/raw foods) are more likely to develop an unhealthy body composition and DIM, which in turn, may promote progression of obesity to MetS. A nutritional iron deficiency occurs when an insufficient amount of dietary iron is absorbed to meet the requirements of the body. Although a Western diet is rich in meat, which contains bioavailable heme iron, it is also associated with increased fat intake. Chang et al. showed that overweight and obese women who consumed a "high-fat, low-carbohydrate" diet had a 10-fold higher risk of developing iron-deficiency anemia (IDA; odds ratio (OR): 10.12; 95\% CI: 1.27-80.79; $p=0.017$ ) [30]. Increased intake of dietary fat but a low intake of steamed/boiled/raw foods (e.g., vegetables) may increase systemic inflammation and promote the pathophysiology of DIM [7,12,17].

The DIM-associated dietary pattern explained $64.7 \%$ of the total variation in food groups and $36.5 \%$ of the total variation in response variables. In this study, we used four response variables (SF, hepcidin, ALT, and HDL-C) because of their strong correlations with DIM. Among these response variables, the DIM-associated dietary pattern was largely explained by SF (which explained $14.6 \%$ of the variation in dietary pattern scores), hepcidin (which explained $8.9 \%$ of the variation), ALT (which explained $8.8 \%$ of the variation), and to a lesser extent, serum HDL (which explained $4.3 \%$ of the variation). Numerous studies have shown that elevated SF concentrations and body iron stores are strong risk factors for visceral fat mass and metabolic disorders [3,26-28]. Moreover, excess adiposity can increase hepcidin secretion, which possibly acts through proinflammatory cytokines or leptin or IR, and elevated serum hepcidin may result in a lower iron absorption rate and hypoferremia $[7,8,17,29]$. 
Our study found that SF was positively associated with the consumption of processed meats ( $\beta=18.65 ; 95 \%$ CI: $0.03-37.27 ; p=0.05$ ) and negatively associated with steamed/boiled/raw food intake ( $\beta=-13.87 ; 95 \%$ CI: $-27.75-0.02 ; p=0.05$ ) (data not shown). Beck et al. found that high intake of red meat, poultry, fish, and vegetables was associated with higher levels of $\mathrm{Hb}$, serum iron, and SF [18]. Avila et al. also reported a positive correlation between red meat intake and SF levels among Chilean men [31]. Our study also found that hepcidin was significantly associated with the consumption frequency of deep-fried foods ( $\beta=24.31 ; 95 \%$ CI: 4.87-43.75; $p=0.015$ ). Deep-fried foods can trigger hepatic hepcidin production via oxidative mediators (e.g., NO) and proinflammatory cytokines (e.g., tumor necrosis factor- $\alpha$ ) [32]. HDL-C was inversely associated with the frequency of eating out ( $\beta$ $=-2.74 ; 95 \%$ CI: $-5.34-0.14 ; p=0.039)$. Cohen and Bhatia showed that eating out was associated with metabolic disorder diseases [33]. Foods purchased away from home tend to be energy-dense and prepared by deep-frying and not steaming/boiling compared to homemade foods [33,34].

Traditionally, a liver biopsy is the gold standard method for diagnosing hepatic iron overload. However, a liver biopsy is invasive and costly and occasionally may cause complications. The World Health Organization (WHO) suggests that, in the absence of HFE and inflammation, SF can serve as a surrogate marker for diagnosing iron overload ( $>200 \mu \mathrm{g} / \mathrm{L}$ in men or $>150 \mu \mathrm{g} / \mathrm{L}$ in women) [35]. However, SF is sensitive to inflammation and might not truly reflect hepatic iron overload. Recently, non-invasive approaches, like magnetic resonance (MR) imaging, are being used for hepatic iron quantification, and it is regarded as a suitable substitute for SF levels and a liver biopsy. Branisso et al. examined 152 biopsy-proven NAFLD patients and found that $37 \%$ patients had DIOS. In addition, those authors showed that the cutoff point of the ferritin level for diagnosing hepatic iron overload using a liver biopsy was $284.3 \mathrm{ng} / \mathrm{mL}$ [36]. In the current study, we used gender-specific cutoff points for diagnosing hyperferritinemia ( $>300 \mathrm{ng} / \mathrm{mL}$ in males and $>200 \mathrm{ng} / \mathrm{mL}$ in females). Based on this definition, the current study found only $55.3 \%$ of DIM patients with hyperferritinemia. We reasoned that the relatively low prevalence rate of hyperferritinemia may have been due in part to the difference in SF cutoff points. Future studies are needed to validate the cutoff point of SF for diagnosing hepatic iron overload in overweight and obese adults.

In the present study, the prevalence rate of DIM was $22.6 \%$ (22.9\% in males and $22.3 \%$ in females). This rate is similar to that reported by Dongiovanni and colleagues [21] that $20 \% \sim 30 \%$ of patients with NAFLD and MetS had DIM. In contrast, Deugnier et al. [8] reported that the prevalences of DIM in patients with NAFLD were $34.5 \%$ and $51.5 \%$, respectively. This discrepancy may have been due in part to the lack of consensus on a clinical definition of DIM. Despite identifying certain clinical features, there is still no clinical cutoff point for a diagnosis of DIM. Deugnier and colleagues proposed diagnostic criteria for DIM based on three criteria: (1) normal or moderately increased TS $(<60 \%)$; (2) the presence of metabolic disorders (high BMI, android distribution of fat, hypertension, dyslipidemia, and high glucose levels); and (3) excessive hepatic iron levels [8]. Dongiovanni et al. proposed that clinical features of DIM be defined by (1) the presence of obesity and metabolic alterations, (2) the presence of a fatty liver, (3) hyperferritinemia with normal or low serum iron, (4) mild increases in hepatic and body iron stores, and (5) the absence of ferroportin-1 mutations or polymorphisms [21]. In the present study, we defined DIM as the presence of at least four of the following criteria: (1) serum hepcidin (>200 ng/mL in men and $>140 \mathrm{ng} / \mathrm{mL}$ in women), (2) hyperferritinemia ( $>300 \mathrm{ng} / \mathrm{mL}$ in men and $>200 \mathrm{ng} / \mathrm{mL}$ in women), (3) central obesity, (4) NAFLD, and (5) the presence of at least two abnormal metabolic profiles. Currently, there is no definitive clinical cutoff point for abnormal values of serum hepcidin. In our study, mean concentrations of serum hepcidin for adult men and women were $184.91 \pm 9.38$ and 115.44 $\pm 10.34 \mathrm{ng} / \mathrm{mL}(p<0.001)$, respectively. Mean serum hepcidin levels for normal-weight men and overweight/obese men were $165.39 \pm 12.38$ and $200.83 \pm 13.47 \mathrm{ng} / \mathrm{mL}(p=0.046)$ and $102.95 \pm 13.06$ and $140.33 \pm 19.69 \mathrm{ng} / \mathrm{mL}(p=0.200)$ for normal-weight and overweight/obese women, respectively. Based on these findings, we defined elevated serum hepcidin according to mean values of overweight/obese adult men and women of $>200$ and $>140 \mathrm{ng} / \mathrm{mL}$, respectively. Overall, despite differences in the 
diagnosis of DIM and the lack of information on liver iron levels, our study agrees with others in which DIM is more likely to be present in obese adults with metabolic disorders [7,8,21,26].

In this study, we used the RRR to derive DIM-associated dietary patterns. The RRR was chosen because RRR-derived dietary patterns are more likely to reflect dietary patterns associated with health outcomes compared to those derived from other dietary pattern analytical methods such as a principal component analysis (PCA) or factor analysis [20,37,38]. Using the RRR, we could determine which dietary patterns were associated with the development of diseases by combining prior information of disease biomarkers and dietary information from other studies [20,38]. Factors in the RRR were derived from both disease-specific biomarkers (responses) and food groups (predictors), while factors obtained from a PCA reflect only dietary habits of the population [39]. In the RRR, we chose serum hepcidin, ferritin, ALT, and HDL as response variables based on their strong correlations with DIM. So, dietary pattern scores were generated by taking into account both the power of predictors (food groups consumed in our study population) and response variables. In contrast, the PCA relies solely on inter-correlations among dietary variables, which might not fully reflect dietary qualities that are most relevant to specific disease etiologies [37]. A PCA or factor analysis was criticized for its subjectivity and for being poorly correlated with disease risks in some studies $[20,40]$.

There are some limitations to this study. First, this was a cross-sectional study; therefore, causal relationships could not be evaluated. Second, there was a relatively small sample size $(n=208)$. Third, hyperferritinemia was used to indicate iron dysregulation and not hepatic iron levels by a liver biopsy. Liver biopsies are the gold standard for assessing tissue iron overload, but it would be difficult to perform them in a population-based study. Although SF is more suitable for population-based studies, obesity-related inflammation might also induce hyperferritinemia. Hence, SF is also regarded as an acute-phase reactant and is associated with a risk of MetS. Fourth, dietary patterns were assessed by the FFQ and not by 24-h dietary recall; therefore, we did not know the actual amount of food intake of participants. Fifth, the RRR was used to derive DIM-associated dietary pattern scores. The RRR method requires in-depth knowledge of diet-disease relationships in order to select appropriate response variables. Our study selected four response variables (hepcidin, ferritin, ALT, and HDL-C) based on their strong correlations with DIM. However, selecting response variables can be subjective, and this might have resulted in different dietary patterns in different age groups or studies.

\section{Conclusions}

Our results suggest that individuals who tend to eat out and that have a preference for a Western diet (indicated by high intake frequencies of meat, coffee, and fatty and deep-fried foods and low intake of steamed/boiled/raw foods) are more likely to develop an unhealthy body composition and DIM, which in turn, may promote the progression of obesity to MetS. Future research with a larger sample size or different populations is required to validate our current findings due to variations in the habitual consumption of different food groups.

Supplementary Materials: The following are available online at http://www.mdpi.com/2072-6643/11/11/2733/s1: Table S1: Strengthening the Reporting of Observational Studies in Epidemiology (STROBE) Statement-Checklist of items that should be included in reports of cross-sectional studies.

Author Contributions: A.R.C. conducted data analyses and drafted the manuscript. S.-H.T. and K.-C.Y. contributed to patient recruitment and reagents/materials/analytical tools. S.-H.T., A.A.T., A.V.S., and C.-H.B. contributed to the study design and statistical analysis. J.-S.C. conceptualized and designed the study, revised the initial manuscript, and approved the final manuscript.

Funding: This research and the APC were funded by Taipei Medical University Hospital (107TMU-TMUH-11 and 108TMU-TMUH-14) and the Ministry of Science and Technology, Taiwan (MOST 107-2320-B-038-010-MY3).

Acknowledgments: Jung-Su Chang was supported by grants from Taipei Medical University Hospital (107TMU-TMUH-11 and 108TMU-TMUH-14) and the Ministry of Science and Technology, Taiwan (MOST 107-2320-B-038-010-MY3).

Conflicts of Interest: The authors declare no conflicts of interest. 


\section{References}

1. Chang, H.C.; Yang, H.C.; Chang, H.Y.; Yeh, C.J.; Chen, H.H.; Huang, K.C.; Pan, W.H. Morbid obesity in Taiwan: Prevalence, trends, associated social demographics, and lifestyle factors. PLoS ONE 2017, 12, e0169577. [CrossRef] [PubMed]

2. Zhao, L.; Zhang, X.; Shen, Y.; Fang, X.; Wang, Y.; Wang, F. Obesity and iron deficiency: a quantitative meta-analysis. Obes. Rev. 2015, 16, 1081-1093. [CrossRef] [PubMed]

3. Chang, J.S.; Lin, S.M.; Huang, T.C.; Chao, J.C.; Chen, Y.C.; Pan, W.H.; Bai, C.H. Serum ferritin and risk of the metabolic syndrome: a population-based study. Asia. Pac. J. Clin. Nutr. 2013, 22, 400-407. [CrossRef] [PubMed]

4. Recalcati, S.; Gammella, E.; Cairo, G. Dysregulation of iron metabolism in cancer stem cells. Free Radic. Biol. Med. 2019, 133, 216-220. [CrossRef] [PubMed]

5. Ginzburg, Y.Z.; Feola, M.; Zimran, E.; Varkonyi, J.; Ganz, T.; Hoffman, R. Dysregulated iron metabolism in polycythemia vera: etiology and consequences. Leukemia 2018, 32, 2105-2116. [CrossRef] [PubMed]

6. Halon-Golabek, M.; Borkowska, A.; Herman-Antosiewicz, A.; Antosiewicz, J. Iron Metabolism of the Skeletal Muscle and Neurodegeneration. Front. Neurosci. 2019, 13, 165. [CrossRef] [PubMed]

7. Aigner, E.; Feldman, A.; Datz, C. Obesity as an emerging risk factor for iron deficiency. Nutrients 2014, 6 , 3587-3600. [CrossRef] [PubMed]

8. Deugnier, Y.; Bardou-Jacquet, E.; Laine, F. Dysmetabolic iron overload syndrome (DIOS). Presse Med. 2017, 46, e306-e311. [CrossRef] [PubMed]

9. Shim, Y.S.; Kang, M.J.; Oh, Y.J.; Baek, J.W.; Yang, S.; Hwang, I.T. Association of serum ferritin with insulin resistance, abdominal obesity, and metabolic syndrome in Korean adolescent and adults: The Korean National Health and Nutrition Examination Survey, 2008 to 2011. Medicine 2017, 96, e6179. [CrossRef] [PubMed]

10. Suarez-Ortegon, M.F.; Ensaldo-Carrasco, E.; Shi, T.; McLachlan, S.; Fernandez-Real, J.M.; Wild, S.H. Ferritin, metabolic syndrome and its components: A systematic review and meta-analysis. Atheroscler. 2018, 275, 97-106. [CrossRef] [PubMed]

11. McKay, A.; Wilman, H.R.; Dennis, A.; Kelly, M.; Gyngell, M.L.; Neubauer, S.; Bell, J.D.; Banerjee, R.; Thomas, E.L. Measurement of liver iron by magnetic resonance imaging in the UK Biobank population. PLoS ONE 2018, 13, e0209340. [CrossRef] [PubMed]

12. Ma, X.; Pham, V.T.; Mori, H.; MacDougald, O.A.; Shah, Y.M.; Bodary, P.F. Iron elevation and adipose tissue remodeling in the epididymal depot of a mouse model of polygenic obesity. PLoS ONE 2017, 12, e0179889. [CrossRef] [PubMed]

13. Sabrina, N.; Bai, C.H.; Chang, C.C.; Chien, Y.W.; Chen, J.R.; Chang, J.S. Serum Iron:Ferritin Ratio Predicts Healthy Body Composition and Reduced Risk of Severe Fatty Liver in Young Adult Women. Nutrients 2017, 9, 833. [CrossRef] [PubMed]

14. Abbaspour, N.; Hurrell, R.; Kelishadi, R. Review on iron and its importance for human health. J. Res. Med. Sci. 2014, 19, 164-174. [PubMed]

15. Girelli, D.; Nemeth, E.; Swinkels, D.W. Hepcidin in the diagnosis of iron disorders. Blood 2016, 127, $2809-2813$. [CrossRef] [PubMed]

16. Rishi, G.; Wallace, D.F.; Subramaniam, V.N. Hepcidin: regulation of the master iron regulator. Biosci. Rep. 2015, 35. [CrossRef] [PubMed]

17. Schmidt, P.J. Regulation of Iron Metabolism by Hepcidin under Conditions of Inflammation. J. Biol. Chem. 2015, 290, 18975-18983. [CrossRef] [PubMed]

18. Beck, K.; Conlon, C.; Kruger, R.; Coad, J. Dietary Determinants of and Possible Solutions to Iron Deficiency for Young Women Living in Industrialized Countries: A Review. Nutrients 2014, 6, 3747-3776. [CrossRef] [PubMed]

19. Quintana Pacheco, D.A.; Sookthai, D.; Wittenbecher, C.; Graf, M.E.; Schubel, R.; Johnson, T.; Katzke, V.; Jakszyn, P.; Kaaks, R.; Kuhn, T. Red meat consumption and risk of cardiovascular diseases-is increased iron load a possible link? Am. J. Clin. Nutr. 2018, 107, 113-119. [CrossRef] [PubMed]

20. Hoffmann, K.; Schulze, M.B.; Schienkiewitz, A.; Nothlings, U.; Boeing, H. Application of a New Statistical Method to Derive Dietary Patterns in Nutritional Epidemiology. Am. J. Epidemiol. 2004, 159, 935-944. [CrossRef] [PubMed]

21. Dongiovanni, P.; Fracanzani, A.L.; Fargion, S.; Valenti, L. Iron in fatty liver and in the metabolic syndrome: a promising therapeutic target. J. Hepatol. 2011, 55, 920-932. [CrossRef] [PubMed] 
22. Vandenbroucke, J.P.; von Elm, E.; Altman, D.G.; Gotzsche, P.C.; Mulrow, C.D.; Pocock, S.J.; Poole, C.; Schlesselman, J.J.; Egger, M.; Initiative, S. Strengthening the Reporting of Observational Studies in Epidemiology (STROBE): explanation and elaboration. PLoS Med. 2007, 4, e297. [CrossRef] [PubMed]

23. Von Elm, E.; Altman, D.G.; Egger, M.; Pocock, S.J.; Gotzsche, P.C.; Vandenbroucke, J.P.; Initiative, S. The Strengthening the Reporting of Observational Studies in Epidemiology (STROBE) Statement: guidelines for reporting observational studies. Int. J. Surg. 2014, 12, 1495-1499. [CrossRef] [PubMed]

24. Ministry of Health and Welfare. 2017 Annual Report of Health Promotion Administration. Available online: https://www.hpa.gov.tw/EngPages/List.aspx?nodeid=1070 (accessed on 7 June 2018).

25. Niu, J.; Seo, D.-C. Central obesity and hypertension in Chinese adults: a 12-year longitudinal examination. Prev. Med. 2014, 62, 113-118. [CrossRef] [PubMed]

26. Datz, C.; Felder, T.K.; Niederseer, D.; Aigner, E. Iron Homeostasis in the Metabolic Syndrome. Eur. J. Clin. Invest. 2013, 43, 215-224. [CrossRef] [PubMed]

27. Utzschneider, K.M.; Largajolli, A.; Bertoldo, A.; Marcovina, S.; Nelson, J.E.; Yeh, M.M.; Kowdley, K.V.; Kahn, S.E. Serum ferritin is associated with non-alcoholic fatty liver disease and decreased B-cell function in non-diabetic men and women. J. Diabetes Complicat. 2014, 28, 177-184. [CrossRef] [PubMed]

28. Kim, T.H.; Hwang, H.J.; Kim, S.H. Relationship between serum ferritin levels and sarcopenia in Korean females aged 60 years and older using the fourth Korea National Health and Nutrition Examination Survey (KNHANES IV-2, 3), 2008-2009. PLoS ONE 2014, 9, e90105. [CrossRef] [PubMed]

29. Vuppalanchi, R.; Troutt, J.S.; Konrad, R.J.; Ghabril, M.; Saxena, R.; Bell, L.N.; Kowdley, K.V.; Chalasani, N. Serum hepcidin levels are associated with obesity but not liver disease. Obesity (Silver Spring) 2014, 22, 836-841. [CrossRef] [PubMed]

30. Chang, J.S.; Chen, Y.C.; Owaga, E.; Palupi, K.C.; Pan, W.H.; Bai, C.H. Interactive effects of dietary fat/carbohydrate ratio and body mass index on iron deficiency anemia among Taiwanese women. Nutrients 2014, 6, 3929-3941. [CrossRef] [PubMed]

31. Avila, F.; Echeverria, G.; Perez, D.; Martinez, C.; Strobel, P.; Castillo, O.; Villaroel, L.; Mezzano, D.; Rozowski, J.; Urquiaga, I.; et al. Serum Ferritin Is Associated with Metabolic Syndrome and Red Meat Consumption. Oxid. Med. Cell. Longev. 2015, 2015, 769739. [CrossRef] [PubMed]

32. Meli, R.; Mattace Raso, G.; Irace, C.; Simeoli, R.; Di Pascale, A.; Paciello, O.; Pagano, T.B.; Calignano, A.; Colonna, A.; Santamaria, R. High Fat Diet Induces Liver Steatosis and Early Dysregulation of Iron Metabolism in Rats. PLoS ONE 2013, 8, e66570. [CrossRef] [PubMed]

33. Cohen, D.A.; Bhatia, R. Nutrition standards for away-from-home foods in the USA. Obes. Rev. 2012, 13, 618-629. [CrossRef] [PubMed]

34. Bozoglu, M.; Bilgic, A.; Yen, S.T.; Huang, C.L. Household food expenditures at home and away from home in Turkey. In Proceedings of the Agricultural \& Applied Economics Association's 2013 AAEA \& CAES Joint Annual Meeting, Washington, DC, USA, 4-6 August 2013.

35. WHO. Serum Ferritin Concentrations for The Assessment of IronStatus and Iron Deficiency in Populations. In Vitamin and Mineral Nutrition Information System; World Health Organization: Geneva, Switzerland, 2011.

36. Branisso, P.P.F.; Oliveira, C.P.; Leão Filho, H.M.; Santos, A.; Lima, F.R.; Mancini, M.; Carrilho, F.J.; Rocha, M.; Cercato, C. Iron-Overload Evaluation by Noninvasive Methods in Patients with Nonalcoholic Fatty Liver Disease, Overweight, and Hyperferritinemia. Diabetes 2018, 67. [CrossRef]

37. Nettleton, J.A.; Steffen, L.M.; Schulze, M.B.; Jenny, N.S.; Barr, R.G.; Bertoni, A.G.; Jacobs, D.R., Jr. Associations between markers of subclinical atherosclerosis and dietary patterns derived by principal component analysis and reduce rank regression in the Multi-Ethnic Study of Atherosclerosis (MESA). Am. J. Clin. Nutr. 2007, 85, 1615-1625. [CrossRef] [PubMed]

38. Weikert, C.; Schulze, M.B. Evaluating dietary patterns: the role of reduced rank regression. Curr. Opin. Clin. Nutr. Metab. Care 2016. [CrossRef] [PubMed]

39. Manios, Y.; Kourlaba, G.; Grammatikaki, E.; Androutsos, O.; Ioannou, E.; Roma-Giannikou, E. Comparison of two methods for identifying dietary patterns associated with obesity in preschool children: the GENESIS study. Eur. J. Clin. Nutr. 2010, 64, 1407-1414. [CrossRef] [PubMed] 
40. Newby, P.; Tucker, K.L. Empirically derived eating patterns using factor or cluster analysis: a review. Nutr. Rev. 2004, 62, 177-203. [CrossRef] [PubMed] 